\title{
REPEATED OVARIOTOMY.*
}

\author{
By J. A. C. KYNOCH, M.B., F.R.C.P. (Edin.), \\ Professor of Midwifery and Gynæcology, St. Andrews University; Obstetric Physician \\ to the Dunclee Royal Infirmary.
}

THE necessity for repeated ovariotomy in the same patient is somewhat rare. Of 228 cases of unilateral ovariotomy performed by Spencer Wells, recurrence in the presumably healthy ovary, necessitating a second operation, was noted in six cases; and of 300 cases collected by Pfannenstiel in the Breslau Klinik, six required a second ovariotomy.

Vellit $z$ has collected $\mathbf{I} 35$ cases of repeated laparotomies for various causes, and in $5^{6}$ of those the indication was cystic disease of the ovary, which was apparently healthy at the first operation.

The interval between the two operations varies, according to Olshausen, from six months to twenty-five years. Most are reported to have taken place within two years, although a much longer period has elapsed in some cases before the second operation was performed. Doran reports two cases of repeated ovariotomy, five and fourteen years after the first operation. Dr. Croom recently reported before this society a case where he removed a large dermoid cyst of the left ovary from a patient eighteen years of age. The right ovary, apparently healthy, was not removed. Eighteen months later the patient was readmitted to hospital on account of recurrent abdominal swelling, which proved, at a second operation, to be due to a papillomatous tumour of the right ovary.

It is of importance in considering the question of recurrent ovarian tumours to exclude those cases where the second tumour had its origin from ovarian tissue left in the pedicle, or from part of the cyst having been left behind at the original incomplete operation. The mortality from the second operation is slightly higher than the first, probably because of greater frequency of adhesions. Olshausen gives the mortality as I2 per cent., whilst Vellitz finds the mortality from the second operation to be as high as 26 per cent.

Repeated ovariotomy raises the interesting question: What is to be done with the apparently healthy ovary in cases of operation for unilateral cystic disease?

Previous to the recent researches upon the pathology of papillomatous ovarian tumours, more especially by Pfannenstiel and

\footnotetext{
* Read before the Edinburgh Obstetrical Society on June I I, I902.
} 
Williams, those cysts were regarded as forming one of the two great groups of ovarian cysts, and their exact clinical significance was a disputed question. Pfannenstiel shows that papillomatous growths may be associated with perfectly simple ovarian cysts, causing implantation and metastasis on the peritoneum, with ascites, the latter being permanently cured, in many cases, on the removal of the tumour. On the other hand, of cystic carcinomas of the ovary he finds one-half papillary in nature, giving rise to true metastasis in the glands, liver, and other organs. It is, therefore, in the case of papillary cysts that the removal, partial or complete, of the other ovary is of most importance, because it is difficult or impossible during the course of an operation to say with any degree of certainty whether we are dealing with a simple or malignant papillomatous tumour. It is imperative for the operator to inform the patient before operation that the other ovary may at the same time have to be removed. This is specially necessary with patients at the child-bearing period. In women at or about the climacteric it would probably be a safe rule to remove the unaffected ovary in every case. In younger patients we have to consider the relative advantages of leaving the ovary, removing it altogether, or partially excising it. By leaving the ovary the absence of the distressing symptoms associated with an artificial menopause, and the possibility of subsequent pregnancy taking place, have to be contrasted with the slight risk which the patient runs of a repeated ovariotomy. According to the statistics of Spencer Wells, only in $2^{\circ} 6$ per cent. of unilateral ovariotomies is the repeated operation necessary. Of 228 ovariotomies performed by him, I20 of the patients subsequently bore children, and only in six cases, as already mentioned, was a second ovariotomy called for. If our patients could be kept under observation after operation, so that early manifestations of recurrence could be detected, there would not be the same danger in leaving the unaffected ovary. But in the case more especially of papillomatous tumours the recurrent growths spread so rapidly that by the time the patients come under observation operation is frequently useless. Veit has reported such a case where a papillomatous ovarian tumour was removed. Nine months later recurrence took place in the opposite ovary, necessitating a second difficult operation. When the ovary shows incipient cystic disease, simple puncture with the knife or cautery, as first performed by Spencer Wells and later by Schroeder and Martin, may be sufficient. Of ten cases of incipient cystic disease so treated by Martin during the course of a unilateral ovariotomy, three of the patients subsequently became pregnant, 
and in no case was a second operation required for recurrent cystic disease. In small adenomatous and dermoid cysts, resection of the ovary has the advantage in younger patients of probably removing the disease and at the same time leaving sufficient ovarian tissue to permit of ovulation and subsequent pregnancy. Mathai reports from Olshausen's Klinik six cases of pregnancy following partial resection of dermoid cysts. Mere resection of a papillary cyst is not so safe. Odehman recently published a case where a large papillomatous cyst was removed from the left side. The right ovary showed incipient papillomatous disease, and was only resected. The disease recurred in this ovary, and when the patient came under observation complete removal of the affected parts was impossible. Pfannenstiel, writing on this subject, states that in all cases of ovarian tumours, which have a tendency to become bilateral-for example, carcinoma, sarcoma, and papillomatous tumours-and in all forms of tumours in patients over forty years of age-the other ovary must be removed at the same time. The three following cases are of collective interest from the fact that in each, within a period of two and a half years, a second ovariotomy was necessary for the removal of a cystic swelling of the ovary, which at the time of the first operation was apparently sufficiently healthy to warrant its being left behind. Individually they are of interest as illustrating recurrence in each case of a different type of tumour.

CASE I.-Mrs. J., forty years of age, came under my care in the summer of 1898 , complaining of pain and abdominal swelling of four months' duration. She had been married twenty-one years; never pregnant; menstruation regular. On examination there was dulness on percussion over the left lower quadrant of the abdomen, reaching as high as the umbilicus. The uterus was small and retroverted. Diagnosis was left-sided ovarian cyst. Ovariotomy being performed, the tumour proved to be a left-sided multilocular cyst. The pedicle was a long one, and was ligatured in the usual way. Beyond some few adhesions to the abdominal wall, the operation was uncomplicated. The right ovary was felt, but not specially inspected, and, as it did not appear to be enlarged, it was not removed. Her recovery was satisfactory. She enjoyed excellent health for the following two years, but again consulted me on December 5, I90o, two years and a half after operation. She now complained of pain and swelling on the right side of the abdomen, gradually increasing for some months previous to being seen. She had a ventral hernia, for which she was chloroformed, with a view of curing it, when a swelling the size of a cocoa-nut was felt to the right of the uterus. This proved to be a simple multilocular ovarian cyst connected with the right ovary. There were no adhesions, the pedicle was secured in the usual way, and the abdominal wound sutured for the relief of the hernia. Two days after operation her temperature rose to $101 \cdot 8^{\circ}$, pulse 124 . She complained of pain in the back and over the right scapula, with rapid and painful breathing and blood-tinged sputum. There was dulness over the lower half of the right chest behind. Ten days later the temperature and 
pulse were normal, and the pneumonic symptoms had subsided. After that she made an uneventful recovery, and was dismissed cured one month after admission to hospital.

CASE 2.-Mrs. R., thirty-four years of age, consulted me in July, I 899 , on account of pain and swelling of the left side of the abdomen of two months' duration. She considered herself four months pregnant. The pain over the swelling was very severe and continuous for a fortnight before her admission to hospital. Her previous health had been good, and she had had three natural full-time confinements. On examination there was felt a cystic swelling the size of a football, and extremely tender to pressure. To the right of the tumour, and reaching as high as a hand's breadth above the symphysis, there was felt a soft swelling, corresponding in size to a four and a half months' pregnant uterus. The diagnosis was four and a half months' pregnancy, complicated with an ovarian cyst. On July 20 ovariotomy was performed. The tumour was found to be a leftsided ovarian cyst, chiefly unilocular, and covered upon its inner wall with abundant papillary growths. There was some localized peritonitis over the tumour, which probably accounted for the pain complained of previous to operation. The pedicle, which was twisted, was ligatured in the usual way, and, as the right ovary appeared to be perfectly normal, it was not removed. On August I9 she was dismissed well. She continued in good health, and had a normal confinement on December 22, forceps being applied at the outlet. She nursed for a few weeks only, and menstruation was subsequently somewhat irregular. On October I5, I900, fifteen months after operation, she was readmitted on account of dysuria of five weeks' duration. On examination, the abdominal cicatrix retained its dark pigmentation acquired during the later months of pregnancy. The uterus was normal in size, and was pushed to the front and left side by a cystic swelling the size of a child's head, occupying the right and posterior fornix. Diagnosis was ovarian cyst of the right ovary. On October I 8 vaginal ovariotomy was performed, and a unilocular papillomatous cyst was removed. The vaginal route was chosen in this case in preference to a repeated abdominal section, on account of the position, size, and mobility of the tumour. She was dismissed well on November 7 .

CASE 3.- -J. A., thirty-two years of age, was admitted to hospital on March I6, I90o, complaining of swelling of abdomen of five months' duration. Menstruation regular; never pregnant. On examination the abdomen was found occupied by a tense cystic swelling, corresponding in size to a full-time pregnancy. Per vaginam no bulging was found in either lateral fornix, but the lower edge of the tumour could be felt through the anterior fornix on pushing it from above. On March 26 ovariotomy was performed for a tumour having all the appearances of a simple multilocular ovarian cyst. The pedicle was secured in the usual way, and, as there were universal adhesions to the peritoneum, the abdominal cavity was irrigated with sterilized water. The right ovary appeared to be unaffected. On April I there were noted signs of pleurisy with effusion over the base of the left chest behind. The chest signs cleared up in the course of a fortnight, and the subsequent progress of the patient was quite satisfactory. She enjoyed good health, and was able to attend her work in a mill for a continuous period of nine months. She was readmitted to my ward on November 20 of last year (a year and nine months after operation) complaining of abdominal swelling of two and a half months' duration, the increase being specially marked during three weeks previous to admission. Menstruation had been irregular since the first operation. On examination the patient was found anæmic and somewhat emaciated, temperature $\mathrm{IOI}^{\circ}$, pulse $\mathrm{I} 20$. There was found a semicystic abdominal swelling, nodular in parts, and rising in the middle line as high as a hand-breadth above the umbilicus. There was complete 
dulness in both iliac regions, and great pain complained of in palpating the tumour. The uterus was retroverted, the sound passed $\frac{1}{2}$ inch beyond the normal, and through the anterior fornix the lower edge of the tumour could be felt. The circumference of the umbilicus was $36 \frac{1}{2}$ inches. The chest appeared to be normal, and the urine was free of albumen. Diagnosis was right-sided ovarian tumour, probably malignant. On November 26 ovariotomy was performed, and there was found a large quantity of blood-tinged ascitic fluid in the abdomen. The soft friable tumour, somewhat adherent posteriorly, was removed through a long abdominal incision. There was no trace of the left ovary. There was felt a nodule the size of a walnut upon the parietal peritoneum, and two smaller ones on the fundus of the uterus. The patient made a perfectly satisfactory recovery from the operation, but the abdominal pain complained of before operation continued uninterruptedly. This was referred chiefly to the peritoneal growth, which increased rapidly in size. A month after operation she had continuous sickness and vomiting, and she died with all the symptoms of peritoneal cancer two months later. No postmortem was obtained.

Of the three cases which I have reported, the first may be looked upon as a coincidence, both tumours being multilocular and simple in nature. Incipient cystic disease may have been present in the ovary which was left at the first operation, where simple puncture might have prevented further growth; but from the age of the patient its complete removal would, I think, have been justifiable. The second case favours Pfannenstiel's suggestion that in all papillomatous ovarian cysts, irrespective of the age of the patient, the other ovary should be removed, although apparently unaffected, on account of the suspicious nature of those tumours, and their great tendency to bilateral growth. The third case is of special interest over and above the other two, because of its bearing on the subject of the relation of proliferating cystomata of the ovary with cancerous disease of other abdominal organs. In the Edinburgh Hospital Reports, 1892 , Professor Simpson, writing upon this subject, reports a case of double ovariotomy for simple multilocular cystomata, associated with carcinoma of the liver. He refers to the statistics of Spencer Wells, who found that of I,000 ovariotomies, where II 7 of the patients had died after recovery from the operation, thirty-two succumbed to malignant disease at an interval of from six weeks to five years after operation. Various explanations have been suggested to account for the greater tendency such patients have to become the subject of malignant disease. Professor Simpson drew attention to the greater liability to subsequent cancer after double as contrasted with unilateral ovariotomy, and from this fact he suggests that some cases might be accounted for by the absence of healthy ovarian products in the system, or to the presence of morbid products entering the circulation from the degenerated organs. 
Olshausen gives two alternatives for this tendency. 'Either,' he says, ' we have to do in the affected cases with a mixed tumour, there being carcinomatous parts present in what appears to be a simple proliferating cyst, or the ovarian tumour was only a myxoid cystoma, but its extirpation gave rise to the carcinoma from some of the epithelial cells of the adenoma having been allowed to remain or fall into the peritoneal cavity. In the latter case these epithelial elements took root in the peritoneum, and were there nourished and developed into carcinomata.'

Pfannenstiel believes that many cases of carcinoma of abdominal organs following on the removal of ovarian tumours are independent of such growth, and are to be accounted for rather by a pre-existing cancerous diathesis. As illustrating this he describes two cases : one where carcinoma of both breasts developed after simple lateral ovariotomy, and one of carcinoma of the stomach occurring a year and four months after the removal of a double papillomatous ovarian tumour.

In the case I report the first tumour, which was removed, appeared to be an ordinary multilocular ovarian cystoma, and in the absence of any suspicion of malignant disease in the abdomen, it was not examined microscopically; nevertheless, it may have contained malignant elements. The cyst which was removed a year and nine months later from the other ovary had also the appearance of a multilocular ovarian cystoma, and microscopic examination of it by Professor Sutherland failed to reveal any cancerous appearances.

Associated with this there were, however, cancerous nodules on the parietal and visceral peritoneum. The origin of these may have been undetected carcinomatous elements in the first tumour removed, or the case may support the suggestion of Professor Simpson that the absence of ovarian products is an element of importance in the causation of abdominal cancer after double ovariotomy.

\section{REFERENCES.}

Veit's ' Handbuch der Gynäkologie,' vol. ii.

Zeitschrift für Gynïk., vol, xxx.

Lancet, vol. ii., I894.

Edin. Obstet. Trans., vol. xxv.

Klinische Vortrïge, vol. iv., 1886.

Zeitschrift fiur Gynïk., vol. xxviii.

Edin. Hosp. Report, I8g2. 